Novel automated measuring system for evaluating labile plasma iron in serum （血清 labile plasma iron（LPI）の新規自動測定系開発）

旭川医科大学大学院医学系研究科博士課程医学専攻

齋藤 豪志

(生田 克哉，畑山 真弓，渋佐 琴恵，松井 康蔵，田中 利輝，土岐 康通，加藤 大介，飯塚 直美，奥村 利勝) 


\title{
Novel automated measuring system for evaluating labile plasma iron in serum
}

\author{
Takeshi Saito', Katsuya Ikuta' D, Mayumi Hatayama', Kotoe Shibusa', Kozo Matsui ${ }^{2}$, \\ Riki Tanaka ${ }^{2}$, Yasumichi Toki', Daisuke Kato ${ }^{2}$, Naomi lizuka² and Toshikatsu Okumura'
}

\begin{abstract}
Background: As the saturation of transferrin by iron in the serum is approximately $30 \%$, iron loaded to the blood can bind to transferrin not bearing iron. Nevertheless, prolonged iron influx finally results in full transferrin saturation, and iron not bound to transferrin will appear in the serum; this iron is known as non-transferrin-bound iron (NTBI). NTBI damages organs through the production of free radicals. Previously, we established an automated quantification system for NTBI; however, measuring labile plasma iron, which is considered as a highly redox-active component of NTBI, should be a better prognostic factor in iron-overloaded patients.

Methods: We designed and developed a novel system for evaluating labile plasma iron utilizing the Trinder reaction. Automated system was utilized because the previously reported methods for labile plasma iron are intricate and the introduction to the clinical stage has been challenging. Validations such as the contribution of serum proteins and metal ions for this system were evaluated using human serum samples.

Results: We confirmed that our novel system can evaluate labile plasma iron utilizing Trinder reaction and the oxidative potential of ceruloplasmin in the serum. This system was also confirmed to be clinically practical. Metals other than iron did not influence this system. We observed that samples with high NTBI did not always exhibit high labile plasma iron and vice versa, highlighting the necessity of labile plasma iron quantification in evaluating the toxicity of NTBI.

Conclusions: Our novel system should contribute to fundamental and clinical research because it can measure labile plasma iron using the high-throughput automated analyser.
\end{abstract}

\section{Keywords}

Labile plasma iron, non-transferrin bound iron, automated quantification system, iron overload, ceruloplasmin

Accepted: 12th June 2019

\section{Introduction}

Although iron is a vital element for almost all living organisms, excess of iron damages various tissues and organs by producing free radicals; thus, the homeostasis of iron must be tightly controlled in the body. ${ }^{1}$ Nevertheless, the iron balance could sometimes collapse in various clinical situations. For example, prolonged or recurrent transfusions of red blood cells could cause iron overload in anaemic patients
'Division of Gastroenterology and Hematology/Oncology, Department of Medicine, Asahikawa Medical University, Asahikawa, Hokkaido, Japan ${ }^{2}$ Research and Development Department, Shino-Test Corporation, Sagamihara, Kanagawa, Japan

\section{Corresponding author:}

Katsuya Ikuta, Division of Gastroenterology and Hematology/Oncology Department of Medicine, Asahikawa Medical University 2-I-I-I Midorigaoka-Higashi, Asahikawa, Hokkaido 078-85 I0, Japan.

Email: ikuta@asahikawa-med.ac.jp 
refractory to other therapies such as thalassaemia, myelodysplastic syndrome, and aplastic anaemia. ${ }^{2-5}$

In the serum, iron is bound to transferrin (Tf), which functions as an iron transporter circulating in the blood and can be used by all cells in the entire body, particularly for erythrocytosis in the bone marrow. Owing to approximately $30 \%$ saturation of $\mathrm{Tf}$ by iron, the loaded iron can bind to $\mathrm{Tf}$, which has not been bound to iron yet, at the beginning. However, in the absence of an active mechanism for iron excretion from the body, prolonged iron influx results in the saturation of $\mathrm{Tf}$, and iron that cannot bound to $\mathrm{Tf}$ appears in the serum; this iron is called as non-Tf-bound iron (NTBI). ${ }^{6}$ Reportedly, NTBI damages organs by readily producing free radicals in the entire body; therefore, the NTBI quantification could be useful for assessing iron toxicity in iron overload. ${ }^{7}$ Previously, we established a novel automated quantification system for NTBI. ${ }^{8}$ Nevertheless, NTBI should be considered as a heterogeneous form of iron, such as albumin-bound iron and citrate-bound iron, and considered that the oxidative potential to produce free radicals is quite different from those forms. ${ }^{9,10}$ Labile plasma iron (LPI) is one of the components that is highly redox active and produces free radicals easily; it is also reported to be clinically relevant for potential iron toxicity and a prognostic factor for survival in transfusiondependent patients. ${ }^{11-15}$ Reportedly, the LPI quantification is technically feasible; however, the previously reported methods are complex, and the introduction to the clinical stage has also been challenging. ${ }^{16,17}$

This study aims to establish a novel automated measuring system that can assess LPI in the serum, using the oxidative activity of ceruloplasmin (CP) in the serum and the Trinder reaction. ${ }^{18,19}$ Our new system could contribute to fundamental and clinical research because it can measure LPI using the high-throughput automated analyser.

\section{Materials and methods}

\section{The measurement principle}

We used the Trinder reaction to measure LPI in the serum. The reaction of ferricyanide with 4-aminoantipyrine (4-AA) and $\mathrm{N}$-(2-carboxyethyl)-N-ethyl- $m$-toluidine (CEMB) hydrochloride induces the reduction of ferricyanide to ferrocyanide with colouring by the oxidative condensation of 4-AA and CEMB, which can be measured by the colorimetric determination. ${ }^{18,19}$ Considering the serum, $\mathrm{Cp}\left(\mathrm{Cu}^{2+}\right)$ should have the highest oxidizing potential compared with other components. ${ }^{20}$ Thus, by adding the serum to this Trinder reaction, $\mathrm{Cp}\left(\mathrm{Cu}^{2+}\right)$ can oxidize ferrocyanide to ferricyanide. Moreover, if there is LPI in the serum sample,
LPI oxidizes $\mathrm{Cp}\left(\mathrm{Cu}^{+}\right)$to $\mathrm{Cp}\left(\mathrm{Cu}^{2+}\right)$, leading to the accelerated absorbance changes that can be detected spectrophotometrically; this reaction should be accelerated only by the oxidative potential of NTBI and not by the NTBI quantity (Figure 1).

\section{Reagents for the automated analysis}

Reagent-1 (R1) comprised the following: potassium ferrocyanide (Kanto Chemical Co., Inc., Tokyo, Japan); 4-AA (Saikyoukasei Corporation, Saitama, Japan); Tris buffer (MP Bio Japan, Tokyo, Japan) and Triton X-100 (Nacalai Tesque, Inc., Kyoto, Japan). Reagent-2 (R2) comprised CEMB hydrochloride (Saikyoukasei Corporation), Tris buffer and Triton X-100. Both reagents were titrated to $\mathrm{pH} 7.0$ and loaded on automated analysers. The final concentrations of the Tris buffer, potassium ferrocyanide, 4-AA and CEMB were settled at 200, 7.5, 0.5 and $0.5 \mathrm{mM}$, respectively. Of note, the reagents can be kept stable for measurements, at least, two months by storing at $-40^{\circ} \mathrm{C}$ (data not shown).

\section{Settings for the automated analyser and assay method}

We measured LPI using a HITACHI 7180 clinical analyser (Hitachi High-Technologies Corporation, Tokyo, Japan) using the following settings: sampling volume: sample/R1/R2 $=10 \quad \mu \mathrm{L} / 150 \quad \mu \mathrm{L} / 50 \quad \mu \mathrm{L}$; assay wavelength: main $/ \mathrm{sub}=550 \mathrm{~nm} / 700 \mathrm{~nm}$; assay method: rate $\mathrm{A}$ and reading point: first $/ \mathrm{last}=24 / 34$ at $37^{\circ} \mathrm{C}$. In the assay method, we first dispensed samples and $\mathrm{R} 1$ into reaction cells on the automated analyser. After mixing and 5-min incubation, R2 was added to the cells, and the mixture was left for 5 min; thus, the total assay time was only $10 \mathrm{~min}$ for each sample. We measured an increase in absorbance at $550 \mathrm{~nm}$, suggesting the presence of LPI in the serum sample. In the experiments using blood samples obtained from humans, the serum was selected as an acceptable sample type.

\section{Serum samples}

We purchased human pooled serum samples without any personal information from Access Biologicals LLC (Vista, CA). In addition, individual human samples without any personal information were purchased from Trina Bioreactives AG (Grabenstrasse, Switzerland). In the pooled serum, serum iron measured by the assay kit (QuickAuto Neo Fe, ShinoTest Corporation, Tokyo, Japan) was $19 \mu \mathrm{M}$ and NTBI values measured by the automated analyser following our previous report were $0.4 \mu \mathrm{M}$, respectively. 


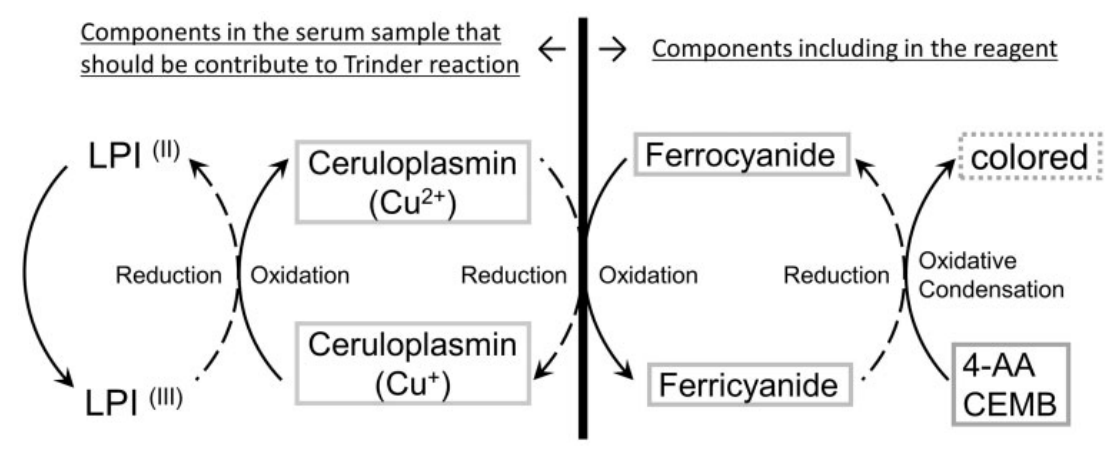

Figure I. The reaction scheme of automated labile plasma iron (LPI) assay system. In this system, the Trinder reaction was used; the reaction of ferricyanide with 4-aminoantipyrine (4-AA) and $\mathrm{N}$-(2-carboxyethyl)- $\mathrm{N}$-ethyl-m-toluidine (CEMB) hydrochloride induces the reduction of ferricyanide to ferrocyanide, which can be measured by the colorimetric determination. By adding the serum to the Trinder reaction, $\mathrm{CP}_{\mathrm{P}}\left(\mathrm{Cu}^{2+}\right)$ can oxidize ferrocyanide to ferricyanide. If there is $\mathrm{LPI}$ in the serum, $\mathrm{LPI}$ oxidizes $\mathrm{CP}_{\mathrm{P}}\left(\mathrm{Cu}^{+}\right)$to $\mathrm{CP}_{\mathrm{P}}\left(\mathrm{Cu}^{2+}\right)$, resulting in the accelerated absorbance changes.

\section{The contribution of iron to the assay}

We added ammonium iron sulphate (Kanto Chemical Co., Inc.) to the pooled human serum samples and measured absorbances indicating LPI. In addition, we added $500 \mu \mathrm{M}$ nitrilotriacetic acid (NTA; Dojindo Laboratories, Kumamoto, Japan) or $500 \mu \mathrm{M}$ ethylene diamine tetraacetic acid (EDTA; Sekisui Medical Co., Ltd, Tokyo, Japan) to R1 and also determined the absorbances. The concentrations of chelators were at least 33 times higher than those of iron in all samples.

\section{The contribution of $C p$ or Tf to the Trinder reaction}

To ascertain the contribution of $\mathrm{Cp}$ in the serum sample for the Trinder reaction, we filtrated the pooled human serum using Amicon ${ }^{\circledR}$-Ultra (Merck KGaA, Darmstadt, Germany) to eliminate endogenous $\mathrm{Cp}$ and $\mathrm{Tf}$ contained in the serum and added different concentrations of Cp (Sigma-Aldrich Japan, Tokyo, Japan) or holo-Tf followed by LPI measurement. In addition, the influence of copper was determined by adding copper chloride (Kanto Chemical Co., Inc.) instead of $\mathrm{Cp}$.

\section{Effect of various transition metals other than iron on the assay}

We added various transition metals, such as copper $(\mathrm{Cu})$, zinc $(\mathrm{Zn})$, magnesium $(\mathrm{Mg})$, cobalt $(\mathrm{Co})$, manganese $(\mathrm{Mn})$, nickel $(\mathrm{Ni})$ and lead $(\mathrm{Pb})$, to the pooled human serum. The final concentrations were settled as $40 \mu \mathrm{M}$, but only $\mathrm{Mg}$ was settled as $2,000 \mu \mathrm{M}$ considering the reference range of the human serum. We purchased all reagents for metals from Kanto Chemical Co., Inc.

\section{Coefficient of variations (CVs) evaluation by intra- and inter-assay}

The intra-assay $\mathrm{CV}$ was assessed using serum samples with 0.67-6.18 $\mu \mathrm{M}$ NTBI. Each sample was applied in 20 replicates in the same run. The inter-assay $\mathrm{CV}$ was determined using samples added with iron to be 6,23 and $46 \mu \mathrm{M}$; all measurements were performed in three replicates for seven days.

\section{Limit of blank and limit of detection}

We defined limit of blank (LOB) and limit of detection (LOD) as follows: $\mathrm{LOB}=$ mean blank $+1.645 \times($ S.D. blank) and $\mathrm{LOD}=\mathrm{LOB}+1.645 \times(\mathrm{S} . \mathrm{D}$. low concentration sample), respectively, where mean blank and S.D.blank are the mean and S.D. of six blank samples assayed in three replicates for five days, and S.D. low concentration sample is the S.D. of 10 low concentration samples assayed in three replicates for five days.

\section{The linearity of the assay}

We assessed the linearity of the assay by measuring serially diluted pooled serum samples added with ammonium iron sulphate as the iron concentration to be $36 \mu \mathrm{M}$.

\section{Comparison with other LPI measuring systems}

The LPI measurement system FeROS ${ }^{\mathrm{TM}}$ LPI Kit (Aferrix Ltd, Kiryat Atidim, Israel) was selected for comparison with our novel method. The LPI measurement was performed per the manufacturer's instructions. Twenty individual serum samples were used for this experiment. 


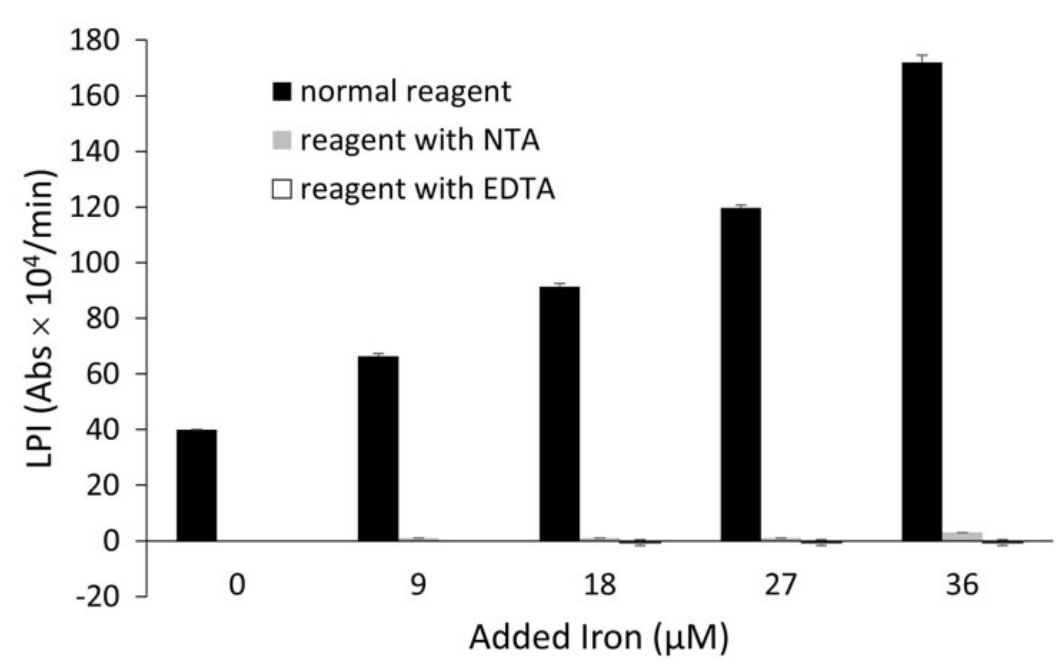

Figure 2. Iron loading to the pooled human serum increased labile plasma iron (LPI). Adding ethylene diamine tetraacetic acid (EDTA) or nitrilotriacetic acid (NTA) to RI cancelled the increase of the absorbance by iron supplementation. The data are represented as the mean $\pm S D$ (the result was representative of two independent experiments $(n=3)$ ).

\section{Correlation between NTBI and LPI}

We measured 100 individual serum samples for NTBI and LPI. Of note, NTBI was measured using the automated analyser developed by us.

\section{Statistical analyses}

All statistical analyses were performed with EZR version 1.38 (Saitama Medical Center, Jichi Medical University, Saitama, Japan), which is a graphical user interface for $\mathrm{R}$ (The $\mathrm{R}$ Foundation for Statistical Computing, Vienna, Austria). This is a modified version of $\mathrm{R}$ commander designed to add statistical functions frequently used in biostatistics. ${ }^{21}$

\section{Results}

The contribution of iron to the established LPI assay

Adding iron to the pooled human serum up to $36 \mu \mathrm{M}$ seemingly increased LPI. When EDTA, which can chelate both copper included in Cp and NTBI, was added to $\mathrm{R} 1$, an increase in the absorbance by iron supplementation was cancelled. Furthermore, when NTA, which can chelate only NTBI, was added to R1, an increase in the absorbance by iron supplementation was cancelled (Figure 2).

The contribution of $C P$ in the serum to the LPI assay

After the removal of proteins, such as $\mathrm{Cp}$ and Tf, in the serum by ultrafiltration, LPI was decreased to almost 0 (Figure 3(a)). When $\mathrm{Cp}$ was added, LPI increased with an increase in the $\mathrm{Cp}$ concentration (Figure 3(b)). The addition of copper ion $\left(\mathrm{Cu}^{2+}\right)$ as copper chloride did not change LPI, however, meaning $\mathrm{Cp}$, rather than $\mathrm{Cu}$, should accelerate the present LPI measurement system (Figure 3(c)). Although Tf contains iron, the addition of $\mathrm{Tf}$ did not increase LPI at all (Figure 3(d)).

\section{Trinder reaction was not accelerated by loading metals other than iron}

In this study, several transitional metals were added to the pooled human serum to determine the contribution of other metals to this assay system. Metals such as $\mathrm{Zn}$, $\mathrm{Mg}, \mathrm{Co}, \mathrm{Mn}, \mathrm{Ni}$ and $\mathrm{Pb}$ did not change LPI (Figure 4 (b) to (g)), although $\mathrm{Cu}$ slightly increased LPI (Figure 4(a)).

\section{The linearity of the LPI assay}

The LPI of the iron-loaded pooled serum measured by this assay system increased linearly (Supplemental Figure 1).

\section{$\angle O B$ and $L O D$ of the $L P I$ assay}

The LOB and LOD of the automated assay system were evaluated as 0.89 and 1.67 , respectively (data not shown). As the mean value LPI of the pooled serum was 34 , the LOD seemed to be adequate to measure LPI. As four different samples were used to measure LPI, 20 times each, the CV values were evaluated to be $\leq 3 \%$ in all samples (data not shown).

\section{Comparison of this novel method with LPI measuring system using ELISA}

We assessed the correlation between our present measuring system and the kit obtained from Aferrix Ltd; 

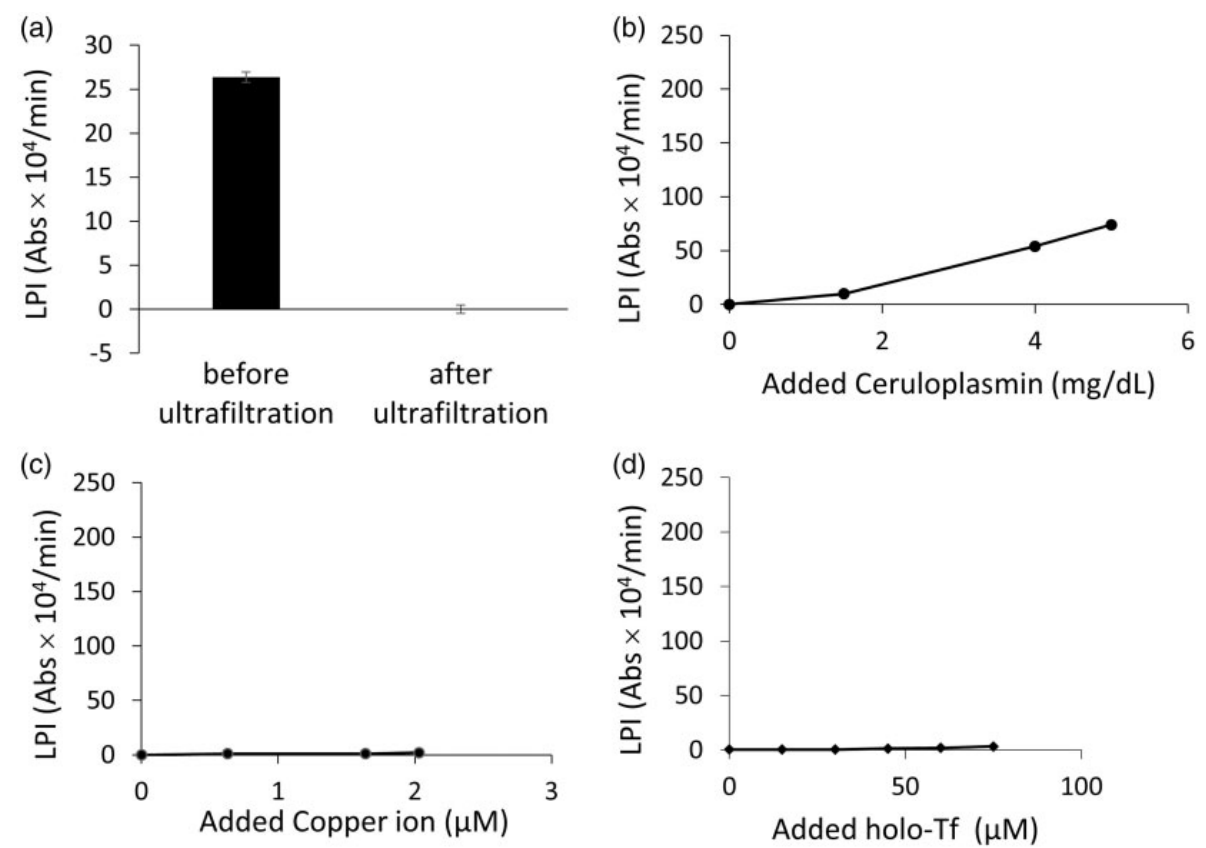

Figure 3. The removal of proteins such as ceruloplasmin (CP) and transferrin (Tf) in the serum by ultrafiltration decreased labile plasma iron (LPI) to almost zero. The data are represented as the mean \pm SD (the result was representative of five independent experiments $(n=20))(a)$. The addition of $C_{p}$ to the ultrafiltrated serum increased LPI with an increase in the Cp concentration $(n=3)(b)$. The addition of copper ion $\left(\mathrm{Cu}^{2+}\right)$ as copper chloride did not change LPI (the result was representative of two independent experiments $(n=3))$ (c). The addition of holo-Tf did not increase LPI $(n=3)(d)$.
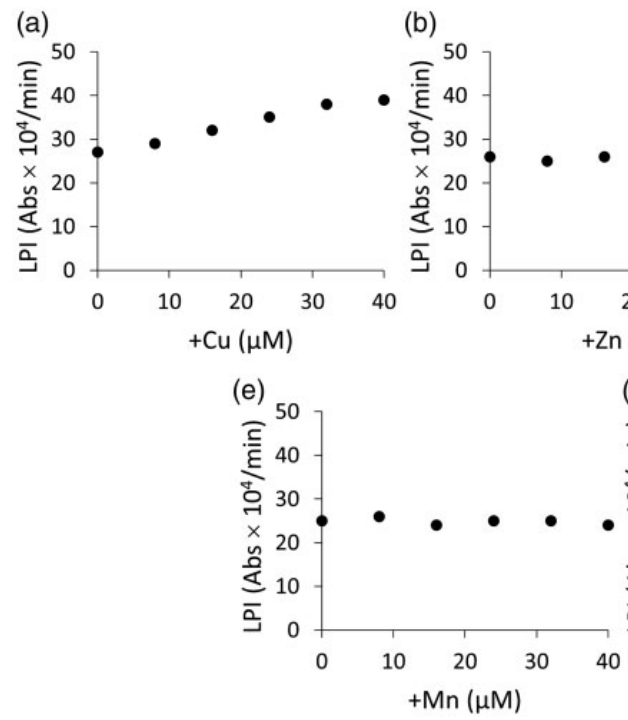

(b)
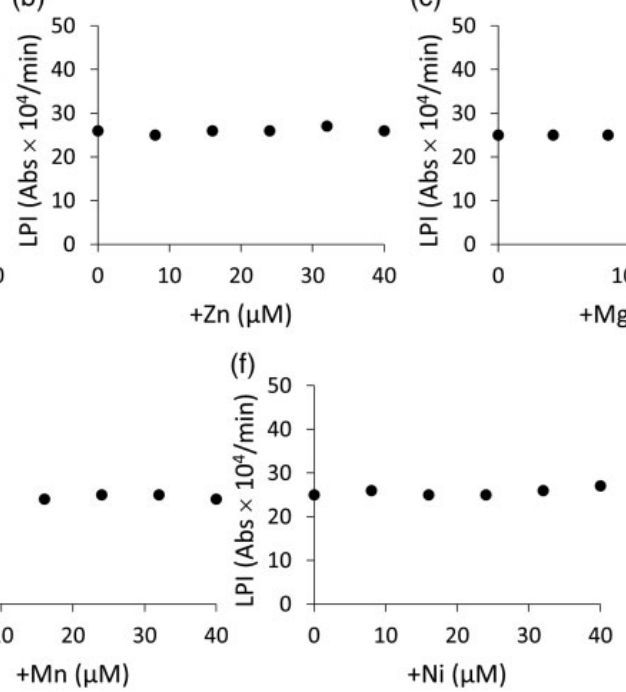

(c)

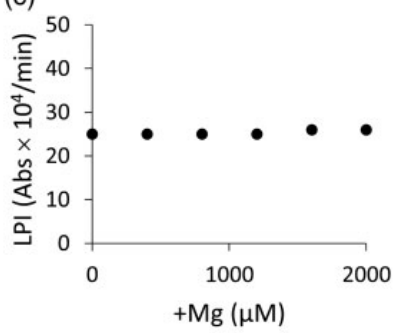

(d)

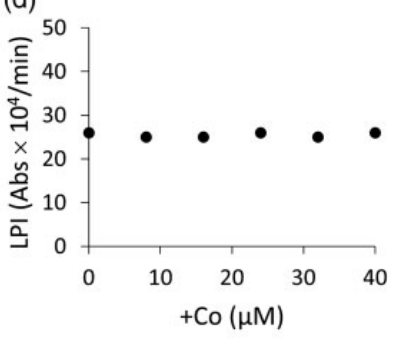

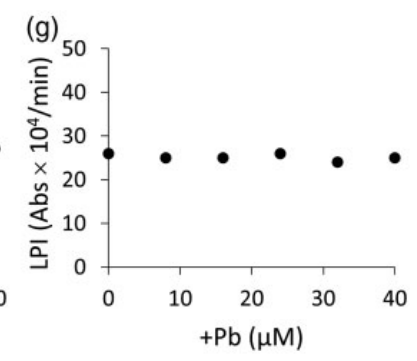

Figure 4. The contributions of metals to this assay system. Although $\mathrm{Cu}$ (a) marginally increased labile plasma iron (LPI), other metals such as $\mathrm{Zn}(\mathrm{b}), \mathrm{Mg}$ (c), Co (d), Mn (e), Ni (f), and Pb (g) did not change LPI. The result was representative of two independent experiments $(n=3)$.

however, we observed some dissociation between the values measured by those two methods (Figure 5). At low LPI concentrations, LPI measured by our system seemingly exhibited partially high values compared with the values determined by the Aferrix system. Alternatively, LPI measured by our system displayed partially low values compared with that measured by the Aferrix system at the higher concentrations of LPI. 


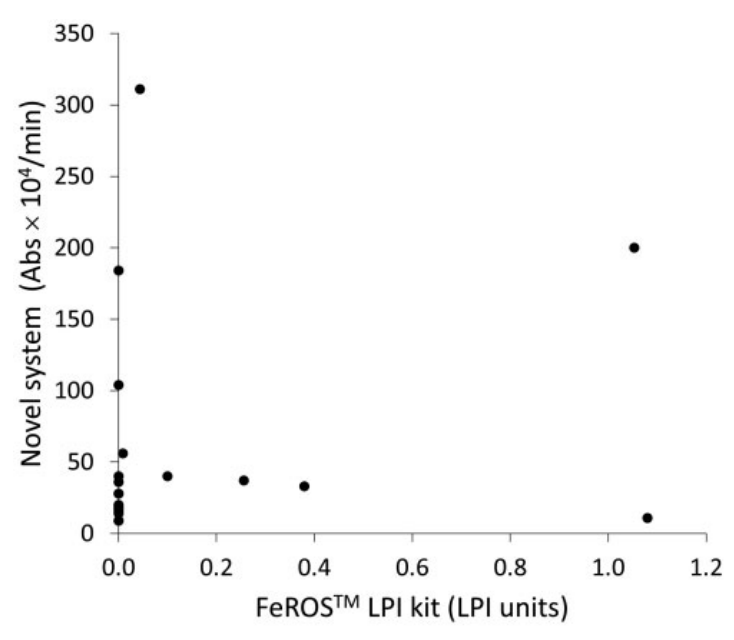

Figure 5. Labile plasma iron (LPI) measured by our novel system and the FeROS ${ }^{\text {TM }}$ LPI Kit (Aferrix Ltd).

\section{Correlation between NTBI and LPI}

In this study, NTBI values measured by our previously established measuring system were compared with LPI values measured by our novel LPI measuring system. The correlation coefficient was 0.3695 , suggesting no strong correlation between NTBI and LPI. For example, some samples exhibited high LPI despite low NTBI (Figure 6(a)). Further analysis concerning the interaction between NTBI and LPI was performed by dividing the samples into three groups: NTBI $=0$ (NTBI-low), $0<\mathrm{NTBI}<0.2$ (NTBI-intermediate) and $0.2 \leq \mathrm{NTBI}$ (NTBI-high). The calculated $P$-value by nonparametric analysis with Kruskal-Wallis test was 0.856 , which indicates that there was no statistical relationship between NTBI and LPI (Figure 6(b)).

\section{Discussion}

The novel method proposed in this study should be considered to measure LPI in the serum samples by using the Trinder reaction. The Trinder reaction in this system involved the oxidative condensation of 4-AA and CEMB under the presence of peroxidase (POD), which can quantify fairly small amount of $\mathrm{H}_{2} \mathrm{O}_{2}$; reportedly, the oxidative potential of $\mathrm{H}_{2} \mathrm{O}_{2}$ can progress this Trinder reaction by POD. ${ }^{18,19}$ In the novel measuring system, ferrocyanide was oxidized to ferricyanide, resulting in the direct colorimetric quantification of the total oxidative potential that the serum samples possessed. Of note, the reagents used in this system did not contain any material that could induce colouring by the Trinder reaction, implying that there should be no blank reaction. Considering the serum, we assumed that $\mathrm{Cp}\left(\mathrm{Cu}^{2+}\right)$ in the serum could be a candidate possessing potent oxidative potential that could oxidize ferrocyanide to ferricyanide. ${ }^{20}$ In this study, the samples from which the fraction of proteins were removed by ultrafiltration did not display colouring in the novel system (Figure 3(a)), suggesting that some proteins, such as $\mathrm{Cp}$ or $\mathrm{Tf}$, could be involved in the colouring reaction. Hence, $\mathrm{Cp}$ was added to ultrafiltrated serum samples, resulting in the dose-dependent colouring, suggesting that $\mathrm{Cp}$ possesses the oxidative potential for converting ferrocyanide to ferricyanide (Figure 3(b)). Conversely, the addition of copper ion $\left(\mathrm{Cu}^{2+}\right)$ as copper chloride did not change LPI, suggesting that $\mathrm{Cu}$ itself could not accelerate the reaction in the novel LPI measurement system (Figure 3(c)). As this study aimed to measure the oxidative potential of iron, the assessment of adding Tf, which binds to iron, was also performed. As a result, adding $\mathrm{Tf}$ did not cause any colouring by Trinder reaction, indicating that Tf-bound iron did not possess oxidative potential that oxidizes ferrocyanide to ferricyanide (Figure 3(d)).

Furthermore, $\mathrm{Cp}\left(\mathrm{Cu}^{2+}\right)$ in the serum sample could oxidize ferrocyanide to ferricyanide, but $\mathrm{Cp}\left(\mathrm{Cu}^{2+}\right)$ itself would be reduced, suggesting the occurrence of the Trinder reaction only once and not being proceeded as a cycle reaction. The reaction time course demonstrated that the absorbances continuously increased by adding iron under the presence of $\mathrm{Cp}$. In this study, the time-course analysis was also performed by adding metal chelators, such as EDTA and NTA, to determine whether iron contributed to the sequential progression of the Trinder reaction. Seemingly, the addition of EDTA and NTA almost completely cancel the increment of absorbances, suggesting that the redox-active metal ion could be involved in the sequential conversion of $\mathrm{Cp}$ between $\mathrm{Cp}\left(\mathrm{Cu}^{2+}\right)$ and $\mathrm{Cp}\left(\mathrm{Cu}^{+}\right)$form. Of note, EDTA removes not only NTBI but also other metals such as $\mathrm{Cu}$; however, NTA cannot remove $\mathrm{Cu}$ from $\mathrm{Cp}$ but removes NTBI, suggesting the likelihood of the specific involvement of NTBI (Figure 2). In addition, we examined the likelihood of the impact of various transition metals, along with metals that are present in the serum with relatively high concentrations and could transfer electrons. Adding $\mathrm{Cu}$ marginally increased the absorbance, but the addition of other metals did not affect the absorbances (Figure 4(a) to (g)); this marginal increase in the absorbance could be attributed to the presence of $\mathrm{Cp}$ in the samples in this experiment. Perhaps, inactive form of $\mathrm{Cp}$ changed to the active form by the binding of $\mathrm{Cu}$ (Figure 4(a)); conversely, LPI was not increased by the addition of $\mathrm{Cu}$ to ultrafiltrated serum samples in which $\mathrm{Cp}$ was removed (Figure 3(c)).

Hence, our novel system could potentially measure the oxidative potential primarily originated from iron not bound to Tf. Thus, we believe that our method could measure 'LPI', although other factors, such as 

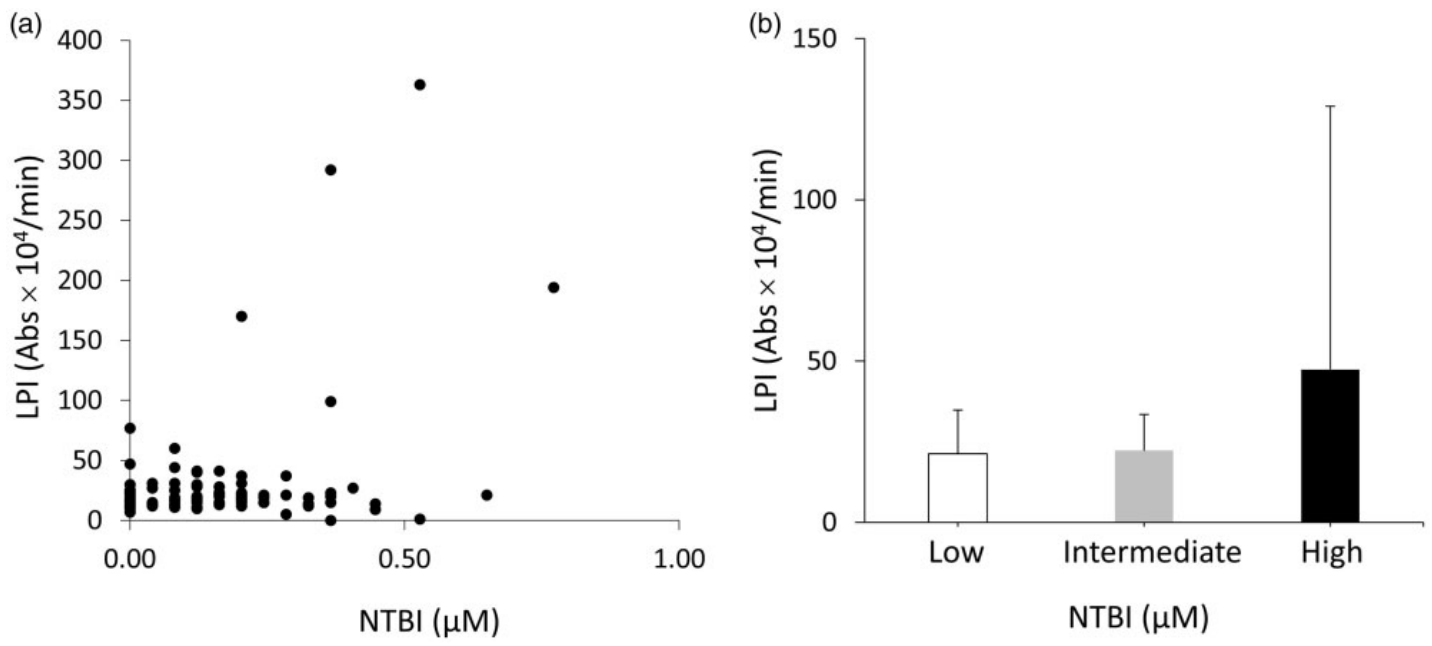

Figure 6. The comparison of labile plasma iron (LPI) measured by our novel system and non-transferrin-bound iron (NTBI) measured by our previously established system. The correlation coefficient was 0.3695 , implying no strong correlation between LPI and NTBI (a). The interaction between NTBI and LPI was also evaluated with non-parametric Kruskal-Wallis test by dividing the samples into three groups: $\mathrm{NTBI}=0$ (Low) (shown as white bar), $0<\mathrm{NTBI}<0.2$ (Intermediate) (shown as grey bar), and $0.2 \leq \mathrm{NTBI}$ (High) (shown as black bar). Calculated P-value was 0.856, indicating that there was no statistical relationship between NTBI and LPI. The data are represented as the mean $\pm S D(b)$.

the $\mathrm{Cp}$ concentration, affect the absorbance. In this study, we also compared this method with other previously established methods measuring LPI. However, no apparent positive correlation was observed when our system was compared with the FeROS ${ }^{\mathrm{TM}}$ LPI Kit (Figure 5); this discrepancy could be attributed to an entirely different approach to measure LPI. FeROS ${ }^{\mathrm{TM}}$ LPI Kit measures LPI as oxidative potential originated from iron itself, whereas our system measures the oxidative potential of iron by using $\mathrm{Cp}$ in serum samples. Thus, our system could be considered to measure the oxidative potential of LPI including the impact of other materials, such as $\mathrm{Cp}$, in each serum sample, although the majority of oxidative stress must be due to LPI. Hence, the determination ranges could also be different, making it difficult to acquire identical results, thereby necessitating further investigation using many clinical samples. One of the potential merits is that our reagent is designed for an automated analyser, implying the feasibility of fast, easy and inexpensive measurement of quite many samples.

Moreover, we assessed the performance and accuracy of our novel method; the linearity was confirmed and validation data, such as $\mathrm{CV}$, seemed to be clinically practical (Supplemental Figure 1). The significance or efficacy of measuring LPI must be assessed with many clinical samples obtained from various diseases or conditions, and our novel system could contribute to this purpose.

The correlation coefficient between NTBI and LPI values measured by our novel measuring system was
0.3695, indicating no strong correlation between them. In this study, some samples exhibited high LPI despite low NTBI and vice versa (Figure 6(a)). Further additional analysis for determining the interaction between NTBI and LPI with non-parametric Kruskal-Wallis test by dividing the samples into three groups such as $\mathrm{NTBI}=0, \quad 0<\mathrm{NTBI}<0.2$ and $0.2 \leq \mathrm{NTBI}$ showed $P$-value of 0.856 , also indicating that there was no statistical relationship between NTBI and LPI (Figure 6(b)). NTBI was present in heterogeneous forms. Although albumin- and citrate-bound NTBI should be considered as the primary forms, unfortunately, little information is available about the heterogeneity of NTBI in each sample, clinical situations or the impact of various substances' concentrations included in the serum. Thus, the correlation between the quantity of NTBI and LPI should be further clarified; this should also highlight the clinical significance of measuring LPI in various clinical situations such as iron overload. We believe our novel LPI measuring system is highly valuable for those studies because it was constructed to measure the oxidative potential primarily derived from LPI and the system's automated methods possess high ability to process huge numbers of samples cost-effectively in short timeframe.

\section{Conclusion}

In summary, we established a novel LPI measuring system. This system should contribute to fundamental and clinical research concerning the iron toxicity 


\section{because it can measure LPI using the high-throughput automated analyser.}

\section{Acknowledgements}

None.

\section{Declaration of conflicting interests}

The authors declared no potential conflicts of interest with respect to the research, authorship, and/or publication of this article.

\section{Funding}

The author(s) disclosed receipt of the following financial support for the research, authorship, and/or publication of this article: TS, KI, YT, MH, TO (Division of Gastroenterology and Hematology/Oncology, Department of Medicine, Asahikawa Medical University) received research funding from Shino-Test Corporation; this study was also performed in collaboration with Shino-Test Corporation.

\section{Ethical approval}

This study is approved by the ethical committee of Asahikawa Medical University (approval number: 18042). (In this article, all data were obtained using only purchased human serum without any personal information.)

\section{Guarantor}

KI.

\section{Contributorship}

TS, KI, MH, KS, KM, RT, YT, DK, NI designed and performed the experiments. TS and KI mainly prepared the article and all co-authors approved the article. TO supervised this study and finally approved the article.

\section{ORCID iD}

Katsuya Ikuta (D) https://orcid.org/0000-0002-7903-5366

\section{Supplemental material}

Supplemental material is available for this article online.

\section{References}

1. Hentze MW, Muckenthaler MU, Galy B, et al. Two to tango: regulation of mammalian iron metabolism. Cell 2010; 142: 24-38.

2. Takatoku M, Uchiyama T, Okamoto S, et al. Retrospective nationwide survey of Japanese patients with transfusion-dependent MDS and aplastic anemia highlights the negative impact of iron overload on morbidity/mortality. Eur J Haematol 2007; 78: 487-494.

3. Suzuki T, Tomonaga M, Miyazaki Y, et al. Japanese epidemiological survey with consensus statement on Japanese guidelines for treatment of iron overload in bone marrow failure syndromes. Int J Hematol 2008; 88: $30-35$.
4. Malcovati L, Della Porta MG and Cazzola M. Predicting survival and leukemic evolution in patients with myelodysplastic syndrome. Haematologica 2006; 91: 1588-1590.

5. Porter JB and Garbowski M. The pathophysiology of transfusional iron overload. Hematol Oncol Clin North Am 2014; 28: 683-701.

6. Hershko C, Graham G, Bates GW, et al. Non-specific serum iron in thalassaemia: an abnormal serum iron fraction of potential toxicity. Br J Haematol 1978; 40: 255-263.

7. Gosriwatana I, Loreal O, Lu S, et al. Quantification of non-transferrinbound iron in the presence of unsaturated transferrin. Anal Biochem 1999; 273: 212-220.

8. Ito S, Ikuta K, Kato D, et al. Non-transferrin-bound iron assay system utilizing a conventional automated analyzer. Clin Chim Acta 2014; 437: 129-135.

9. Grootveld M, Bell JD, Halliwell B, et al. Non-transferrin-bound iron in plasma or serum from patients with idiopathic hemochromatosis. Characterization by high performance liquid chromatography and nuclear magnetic resonance spectroscopy. J Biol Chem 1989; 264: 4417-4422.

10. Hider RC. Nature of nontransferrin-bound iron. Eur J Clin Invest 2002; 32: $50-54$.

11. Koren A, Fink D, Admoni O, et al. Non-transferrin-bound labile plasma iron and iron overload in sickle-cell disease: a comparative study between sickle-cell disease and beta-thalassemic patients. Eur J Haematol 2010; 84: 72-78.

12. Garbowski MW, Evans P, Vlachodimitropoulou E, et al. Residual erythropoiesis protects against myocardial hemosiderosis in transfusion-dependent thalassemia by lowering labile plasma iron via transient generation of apotransferrin. Haematologica 2017; 102: 1640-1649.

13. Gu S, Xv Y, Fei C, et al. Labile plasma iron, more practical and more sensitive to iron overload in myelodysplastic syndromes. Hematology 2017; 22: 9-15.

14. Wood JC, Glynos T, Thompson A, et al. Relationship between labile plasma iron, liver iron concentration and cardiac response in a deferasirox monotherapy trial. Haematologica 2011; 96: 1055-1058.

15. de Swart L, Hendriks JC, van der Vorm LN, et al. Second international round robin for the quantification of serum non-transferrin-bound iron and labile plasma iron in patients with iron-overload disorders. Haematologica 2016; 101: 38-45.

16. Esposito BP, Breuer W, Sirankapracha P, et al. Labile plasma iron in iron overload: redox activity and susceptibility to chelation. Blood 2003; 102: 2670-2677.

17. Cabantchik ZI. Labile iron in cells and body fluids: physiology, pathology, and pharmacology. Front Pharmacol 2014; 5: 45

18. Trinder P. Determination of glucose in blood using glucose oxidase with an alternative oxygen acceptor. Ann Clin Biochem 1969; 6: 24-27.

19. Barham D and Trinder P. An improved colour reagent for the determination of blood glucose by the oxidase system. Analyst 1972; 97: 142-145.

20. Yazawa N, Ono J, Yoshimura H, et al. Influence of nonspecific reaction on determination of $\mathrm{H}_{2} \mathrm{O}_{2}$ using Trinder reagents. Clin Chim Acta 2005; 360: 52-59.

21. Kanda Y. Investigation of the freely-available easy-to-use software "EZR" (Easy R) for medical statistics. Bone Marrow Transplant 2013; 48: $452-458$. 